\title{
Causes of Isolated Oculomotor Nerve Palsy in Patients Presenting to Eye Outpatient Department at Shree Birendra Hospital.
}

\author{
Anu Gurung ${ }^{1}$, Ramesh Raj Bist ${ }^{1}$, Sagar Rajkarnikar ${ }^{1}$, Ram Shrestha ${ }^{1}$. \\ ${ }^{1}$ Department of Ophthalmology, Shree Birendra Hospital.
}

\begin{abstract}
Introduction: Although oculomotor nerve palsy is associated with significant morbidity. The etiology of oculomotor nerve palsy are ischemic, trauma, aneurysm, tumors, inflammatory, idiopathic etc. The present study was performed to determine the etiology of isolated oculomotor nerve palsy in our population.

Methods: The hospital data of patients of isolated oculomotor nerve palsy cases reported to the eye department of Shree Birendra Hospital from March 2009 to March 2011 were collected and retrospectively analyzed for the etiology of the disease. The detail history (ocular and medical), examination and investigations were collected from patient's case records. All patients had been evaluated for hypertension and diabetes mellitus. Neuroimaging, MRI had been performed in all cases. The etiology of isolated oculomotor nerve palsy was classified into ischemia, idiopathic, trauma, aneurysm and neoplastic.
\end{abstract}

Results: During the study period there were 16 eyes of 16 patients who were suffering from isolated oculomotor nerve palsy. The commonest cause was found to be ischemic in 7 patients (43.75\%) followed by idiopathic in 5 patients (31.25\%). Pupil was involved in 3 patients (18.75\%). MRI was carried out in all 16 patients, but revealed positive only in two cases (one intracranial aneurysm and otherNon Hodgkin's Lymphoma). Remaining 14 MRI were normal.

Conclusions: Oculomotor nerve palsy is an uncommon disease and commonest cause was ischemic followed by idiopathic. The cause should be ruled out as the treatment depends on the cause.

Keywords: diabetes mellitus, etiology, hypertension, idiopathic, oculomotor nerve palsy

\section{INTRODUCTION}

Although oculomotor nerve palsy is less common than sixth and fourth cranial nerve palsies, it is associated with significant morbidity. Patients typically present with blepharoptosis, limitation in eye movement accompanied by exotropia and dilated pupil which poorly reacts to light ${ }^{1}$. Oculomotor nerve palsies may result from lesions anywhere along the anatomic pathway from the nucleus to the muscle ${ }^{2}$. Surgical lesion like aneurysms, trauma, tumors may compresses the superficial papillary fibers of pial blood supply causing pupil involving oculomotor nerve palsy ${ }^{3,4}$.

Most reports have stated ischemia as the most common cause of oculomotor nerve palsy ${ }^{5-8}$. Although the majority of the patients with ischemic oculomotor nerve palsy suffer from diabetes mellitus, other disorders like

\section{Correspondence:}

Lt. Col. Dr. Anu Gurung

Department of Ophthalmology, Shree Birendra Hospital

Kathmandu, Nepal

Email: gurung.anu@gmail.com

Phone: +977-9841311027 
hypertension, atherosclerosis may manifest similarly ${ }^{4}$. The American Academy of Ophthalmology (AAO) recommends that cranial MRI with contrast and MRA (Magnetic Resonance Angiography) with special attention to the circus of Willis be performed for the patients with oculomotor nerve palsy with pupil involvement. The AAO also recommends that oculomotor nerve palsy without pupil involvement be re-evaluated during short intervals to detect pupil dysfunction which mandates further diagnostic examinations.

As the study regarding the etiology of isolated oculomotor nerve palsy has not been carried out at shree Birendra Hospital(SBH) till to date, the present study was performed to determine the etiology of isolated oculomotor nerve palsy reported to eye department of SBH.

\section{METHODS}

The hospital data of patients of isolated oculomotor nerve palsy cases reported to the eye department of Shree Birendra Hospital (SBH) from March 2009 to March 2011 were collected and retrospectively analyzed for the etiology of the disease. The detail history (ocular and medical), examination and investigations were collected from patient's case records. The ocular, general and neurological examination had been carried out. Ocular examination included best corrected visual acuity (Snellen's Chart), extraocular movements, pupillary reaction and fundus evaluation. All patients had been evaluated for hypertension and diabetes mellitus. In patients with confirmed diabetes mellitus or hypertension, oculomotor nerve palsy had been diagnosed as ischemic. The routine investigations had been carried out which included routine blood examination and blood sugar level. Neuroimaging, MRI had been performed in all cases. All patients with isolated oculomotor nerve palsy were included in the study.

\section{RESULTS}

Between March 2009 to March 2011 we found 16 eyes of 16 patients with diagnosis of isolated oculomotor nerve palsy reported to eye department of Shree Birendra Hospital. The age ranged from $15-78$ years with mean age 46.5 years. Male to female ratio was 5:3. All patients had unilateral ocular involvement. Patients had presented to the hospital with duration of symptom from 2 days to 6 months.

The most common symptom was deviation of affected eye in 16 patients (100\%), headache in 10 patients $(62.5 \%)$, ptosis in 6 patients (37.5\%). Other symptoms included diplopia, decreased vision and vomiting.

Seven patients (43.75\%) were already on treatment for hypertension and out of which Two patients also suffered from Diabetes Mellitus. In 5 patients (31.25\%) no specific cause was found. Total two patients $(12.5 \%)$ had history of head injury. One patient $(6.25 \%)$ had intracranial aneurysm and one patient (6.25\%) was a case of Non Hodgkin's Lymphoma with metastasis to brain.

Pupil size was normal in 13 patients $(81.25 \%)$ and mydriatic in 3 patients (20\%). MRI was performed for all 16 patients $(100 \%)$ and revealed an abnormality in 2 patients $(12.5 \%)$ including aneurysm and NHL. The MRI in 14 patients $(87.5 \%)$ was normal.

Table 1. Etiology

\begin{tabular}{|l|c|c|}
\hline Etiology & $\begin{array}{c}\text { Number of } \\
\text { cases }\end{array}$ & $\%$ \\
\hline Ischaemic & 7 & $43.75 \%$ \\
\hline Ideopathic & 5 & 31.25 \\
\hline Head Injury & 2 & $12.5 \%$ \\
\hline Intracranial aneurysm & 1 & $6.25 \%$ \\
\hline $\begin{array}{l}\text { Non Hodgkin } \\
\text { Lymphoma }\end{array}$ & 1 & $6.25 \%$ \\
\hline
\end{tabular}

\section{DISCUSSION}

The purpose of the study was to determine the etiology of isolated oculomotor nerve palsy in the patients presented to eye department of Shree Birendra Hospital. In our study male patients were affected more than female patients in the ratio of 5:3. This is similar to the study done by Tabbasi et al where there was male to female ratio of $17: 11 .^{5}$

In the current study, $43.75 \%$ cases were ischemic in origin. Ischemic cause included hypertension and diabetes mellitus. This is similar to the study conducted by Tabassi et al where $42.8 \%$ cases were due to ischemic cause. ${ }^{5}$ In this study 7 patients (43.75\%) suffered from hypertension and among them 2 patients (12.5\%) also suffered from diabetes mellitus unlike in study of Tabassi et al diabetes mellitus was the underlying cause in $32.1 \% .{ }^{5}$ In the study done by Schultz et al the ischemic cause was $58.82 \%{ }^{9}$ Paralysis of the III, IV or VI cranial nerve was first recognized as clinical entity by Ogle 1866 . The diabetic patients have 10 fold increase in the incidence of cranial nerve palsies, with an incidence of $0.97 \%$ among diabetes compared with an incidence of $0.13 \%$ of non diabetic population. ${ }^{10}$

In the current study idiopathic was second common cause of palsy in 5 patients ( $31.25 \%$ ) which is only $17.8 \%$ in the study of Tabassi et al. ${ }^{5}$ Only 2 patients (12.5\%) had history of head trauma in our study whereas trauma was the second common cause in other studies..$^{5-8}$ One patient (6.25\%) had intracranial aneurysm in this study whereas in the study of Scultz et al aneurysm was present in $11.76 \%{ }^{9}$ 
In the current study, in 1 patient (6.25\%) the cause of isolated oculomotor nerve palsy was NHL. Several cases of isolated oculomotor nerve palsy caused by NHL have also been reported. ${ }^{11-13}$ When isolated oculomotor nerve palsy develops in patients with lymphoma, brain involvement should be considered and even if initial brain imaging reveal negative, follow up examinations should be performed to detect the cause of neurologic signs in patients with lymphoma. ${ }^{11}$

In this study there was no pupillary involvement in ischemic type of oculomotor nerve palsy unlike in other studies where pupil was involved in range of $11 \%-32 \%$ in ischemic variety. ${ }^{5-8}$

Since ischemia has been reported as the most common cause of oculomotor nerve paralysis we believe that for cases of oculomotor nerve palsy with pupil sparing, evaluation and control of underlying disorders such as systemic hypertension and diabetes mellitus should be carried out. However in cases of oculomotor nerve palsy with pupillary involvement MRI is indicated.

\section{CONCLUSIONS}

The commonest cause of isolated oculomotor nerve palsy is ischemic followed by idiopathic. MRI is beneficial only if there is pupillary involvement in cases of Isolated oculomotor nerve palsy.

\section{REFERENCES}

1. American Academy of Ophthalmology. Basic and Clinical Science Course: Neuro-ophthalmology. The Academy. 2002-2003:238-45.

2. Albert and Jakobiec. Principles and Practice of Ophthalmology, second edition, vol. 5, pp39994000.

3. Kanski JJ. Clinical ophthalmology, third edition, Butterworth-Heinemann Ltd. 1994,473.
4. Miller N, Newman J. Walsh \& Hoyt's Clinical Neuro-ophthalmology, $5^{\text {th }}$ edition. Philadelphia; Walters Kluwer Co;1999:pp509-36.

5. Tabassi AR, Dehghani AR, Mosayebi H. Etiology of Oculomotor Nerve Paralysis. Journal of Ophthalmic and Vision Research. 2006;1:1.

6. Richards BW. Cause and prognosis in 4278 cases of oculomotor, trochlear and abducens cranial nerves. Am J Ophthalmol. 1992;113:489-96.

7. Rucker CW. The causes of paralysis of third, fourth and sixth cranial nerves. Am J Ophthalmol. 1996;61:1293-98.

8. Rush JA. Paralysis of cranial nerves III, IV and VI: causes and prognosis in 1000 cases. Arch Ophthalmol. 1981;99:76-9.

9. Schultz KL, Lee AG. Diagnostic yield of the evaluation of isolated third nerve palsy in adults. Can J Ophthalmol. 2007;42(1):110-5.

10. Tu MC, Chang YY, Lin TK. Recurrent Multiple Cranial Neuropathies in a Diabetic Patient. ActaNeurol Taiwan.2010;19:208-10.

11. Choi SM, Kim JT, Lee SH et al. Isolated Oculomotor Nerve Palsy due to Non-Hodgkin's lymphoma Demonstrated by Serial MRI. Chonnam Medical Journal. 2008;44 (2):109-12.

12. Park YM, Cho JH, Cho JY, Huh JS, Ahn JY. NonHodgkin's lymphoma of the sphenoid sinus presenting as isolated oculomotor nerve palsy. World J surg Oncol. 2007;3 (5):86.

13. Manabe Y, Korokawa K, Kashihara K, Abe K. Isolated oculomotor nerve palsy in lymphoma. Neurol Res. 2000;22 (4):348-8. 